\title{
Complex patellofemoral reconstruction leads to improved physical and sexual activity in female patients suffering from chronic patellofemoral instability
}

\author{
Patricia M. Lutz ${ }^{1}$ Philipp W. Winkler ${ }^{1} \cdot$ Marco-Christopher Rupp ${ }^{1} \cdot$ Stephanie Geyer ${ }^{1}$. Andreas B. Imhoff ${ }^{1} \mathbb{D}$. \\ Matthias J. Feucht ${ }^{1,2}$
}

Received: 25 August 2020 / Accepted: 15 October 2020 / Published online: 29 October 2020

(C) The Author(s) 2020

\begin{abstract}
Purpose To analyze postoperative physical and sexual activity as well as Quality of Life (QoL) after complex patellofemoral reconstructions in female patients suffering from chronic patellofemoral instability (PFI).

Methods Female patients aged $>18$ years undergoing complex patellofemoral reconstruction for chronic PFI were included. Complex patellofemoral reconstruction was defined as medial patellofemoral ligament reconstruction (MPFL-R) combined with at least one major bony procedure (distal femoral osteotomy, high tibial osteotomy, and trochleoplasty). Outcome was evaluated retrospectively after a minimum follow-up of 12 months using Tegner activity scale, Banff Patellofemoral Instability Instrument 2.0 (BPII 2.0), EuroQol-5D-3L (EQ-5D-3L), EuroQol Visual analog scale (EQ-VAS), and a questionnaire about sexual activity.

Results A total of 34 females (mean age, $26 \pm 5$ years) with a mean follow-up of $45 \pm 16$ months were included. Seventyseven percent had one major bony correction + MPFL-R and 24\% had at least two major bony corrections + MPFL-R. The re-dislocation rate was 6\%. Median Tegner activity scale improved from 3 (range 0-10) to 4 (range 2-6) (n.s.) and an improved activity level was observed in $49 \%$ of subjects. QoL scores showed an EQ-5D-3L Index Value of $0.89 \pm 0.15$, EQVAS of $80.3 \pm 11.4$, and BPII of $68.3 \pm 19.1$. Thirty-four percent of patients reported restrictions of sexual activities due to PFI preoperatively with an improved sexual function observed in $60 \%$ postoperatively due to less pain, improved mobility, and less apprehension. Postoperative return to sexual activity was $91 \%$, whereof $19 \%$ reported current restrictions of sexual function because of pain and/or limited range of motion.

Conclusion Despite the complexity and invasiveness of complex patellofemoral reconstruction, combined bony procedures and MPFL-R resulted in a low redislocation rate, improved physical activity and QoL comparable to values reported after isolated MPFL-R. Furthermore, sexual activity was improved in $60 \%$ of females with preoperative restrictions.
\end{abstract}

Level of evidence IV.

Keywords Patellofemoral instability $\cdot$ Quality of Life $\cdot$ Return to sports $\cdot$ MPFL $\cdot$ Sexual activity $\cdot$ Sex

The research was performed at the Department for Orthopedic Sports Medicine, Technical University Munich, Germany.

Electronic supplementary material The online version of this article (https://doi.org/10.1007/s00167-020-06340-7) contains supplementary material, which is available to authorized users.

Andreas B. Imhoff

imhoff@tum.de

1 Department for Orthopedic Sports Medicine, Technical University Munich, Ismaninger Str. 22, 81675 Munich, Germany

\section{Introduction}

Patellofemoral instability (PFI) includes recurrent dislocation and subluxation of the patella with the highest prevalence observed in young females $[11,15]$. PFI results in

2 Department of Orthopedics and Trauma Surgery, Medical Center, Faculty of Medicine, Albert-Ludwigs-University of Freiburg, Freiburg, Germany 
significant morbidity and functional impairment with a decreased physical activity level $[6,7,11,15,34,36,43]$.

Given the complex interaction between dynamic muscle action, passive soft tissue restrains, surface geometry of the patellofemoral joint, and limb alignment, PFI is based on a multifactorial pathogenesis $[10,27,46]$. Anatomic risk factors, including trochlear dysplasia, valgus malalignment, torsional deformity, patella alta, and a lateralized position of the tibial tuberosity as well as insufficiency of the medial patellofemoral ligament (MPFL) must be taken into consideration for surgical treatment $[3,10,27,28,47]$. Whereas isolated MPFL reconstruction (MPFL-R) may be appropriate in most patients, complex reconstruction procedures are indicated in patients with additional risk factors [10]. Combined MPFL-R and bony corrections such as realignment osteotomies and trochleoplasty are invasive and technically demanding, but have proven to be effective for the treatment of chronic PFI [13, 24, 26, 38].

Over the last years, research on PFI has mainly focused on functional outcomes $[1,4,13,14,24,26,35,44]$, re-dislocation rates [10, 12, 15, 18, 43], and return to sports [30, $36,45]$. Recently, research concerning Quality of Life (QoL) after MPFL-R has gained more attention [4, 5, 17, 19, 21, 22]. Patient-reported QoL outcome measures are commonly seen as multi-dimensional and can therefore be influenced through a range of domains [23]. To date, however, only limited data are available about QoL after complex patellofemoral reconstruction procedures. Furthermore, chronic PFI may also lead to restrictions of sexual activity in young female patients. However, this aspect of QoL has not been investigated for patients with chronic PFI so far.

Better knowledge of postoperative outcomes concerning physical and sexual activity and QoL after complex patellofemoral reconstruction may help to justify such comprehensive procedures and may improve preoperative patient counseling regarding postoperative expectations.

The purpose of this study was to analyze physical activity, QoL, and sexual activity in female patients after complex patellofemoral reconstructions for chronic PFI. The hypothesis was that complex patellofemoral reconstruction improves physical and sexual activity as well as QoL.

\section{Materials and methods}

This retrospective study was conducted to assess physical and sexual activity, as well as QoL after complex patellofemoral reconstructions in young female patients with chronic PFI. The study was approved by the institutional review board of the Technical University of Munich (575/19 S) and conducted according to the Declaration of Helsinki. All subjects gave their written informed consent to participate in this investigation.
For the purpose of this study, only female patients undergoing complex patellofemoral reconstruction (as defined below) between 07/2014 and 06/2019 for chronic PFI were included. Further inclusion criteria were: age $>18$ years and postoperative follow-up of at least 12 months. Exclusion criteria were: relevant comorbidities (infectious diseases, cancer, severe cardiovascular diseases), relevant previous operations independent of operations on the patellofemoral joint, pregnancy at the time of data collection, lack of German language skills, and missing consent.

Complex patellofemoral reconstruction was defined as MPFL-R with or without tibial tubercle osteotomy (TTO) combined with at least one major bony procedure, including distal femoral osteotomy (DFO), high tibial osteotomy (HTO), and trochleoplasty. Indications for major bony procedures were: valgus malalignment $>5^{\circ}$, internal femoral torsion $>30^{\circ}$, external tibial torsion $>40^{\circ}$, and trochlear dysplasia Types B-D according to the Dejour classification [8]. TTO was indicated in patients with a tibial tubercle-trochlear groove (TT-TG) distance $>25 \mathrm{~mm}$ or patella alta.

\section{Data collection}

Medical records were reviewed to collect patient demographics and details about the medical history and surgery.

Physical activity was evaluated by the Tegner activity scale [48] and questions regarding sports ability and frequency.

Global QoL was assessed using EuroQol-5D-3L (EQ5D-3L) and EuroQol Visual analog scale (EQ-VAS) [16, 41, 42]. The EQ-5D-3L measures health related Quality of Life with regard to the 5 dimensions: mobility, self-care, usual activities, pain/discomfort, and anxiety/depression. Each dimension has 3 levels: no problems, some problems, and extreme problems. The descriptive system of the EQ$5 \mathrm{D}-3 \mathrm{~L}$ produces a 5 -digit health state profile which is then converted to an index value ranging from 0 (death) to 1 (full health). The EQ-VAS records the patient's self-rated health on a visual analog scale with the endpoints "best imaginable health state" and "worst imaginable health state".

Disease-specific QoL was assessed using the Banff Patellofemoral Instability Instrument 2.0 (BPII) [2, 20, 31]. In this score patients mark their answers on a visual analog scale. A higher score reflects a higher QoL [23].

Sexual activity was analyzed with a self-designed questionnaire inspired by Nunley et al. [40], which included questions about sexual restrictions pre- and postoperatively and changes in sexual activity postoperatively (Appendix 1).

\section{Statistical analysis}

Statistical analysis was performed using SPSS software version 25.0 (IBM-SPSS, New York, USA). Continuous 
variables were calculated as mean \pm standard deviation allowing one decimal (except for EQ-5D-3L Index Value, allowing two decimals). Categorical variables were reported as count and percentages allowing no decimal. Normal distribution of all data was evaluated with the Kolmogorov-Smirnov test. Longitudinal dependent samples were computed by the paired Wilcoxon signed-rank test for nonparametric data (Tegner activity scale) or with the Chisquare test (sexual restrictions and patellar dislocations pre-/postoperatively). Correlations were calculated using Pearson's correlation coefficient for parametric data and Spearman's correlation coefficient for nonparametric data allowing three decimals. A $p$ value of less than 0.05 was considered to indicate statistical significance.

A retrospective power analysis revealed that with 34 patients, this study has a power of 0.8 to demonstrate a small to moderate effect of $d=$ mean change/standard deviation of 0.5 in a $t$-test for paired samples. Similarly, to be able to obtain a correlation coefficient that significantly differs from $r=0$ with a true correlation of 0.5 , the power is 0.8 [49].

\section{Results}

A total of 34 female patients were included for detailed analysis. Descriptive statistics of the demographical data and the main parameters are shown in Table 1. MPFL-R $( \pm$ TTO) combined with one major bony procedure was performed

Table 1 Descriptive statistics of the demographical data and main parameters of the total study group

\begin{tabular}{ll}
\hline Variable & Total study group \\
\hline Number of included patients & 34 \\
Follow-up [months] & $45.3 \pm 15.7(12-72)$ \\
Age [years] & $25.9 \pm 5.2(18-42)$ \\
BMI [kg/m $\left.{ }^{2}\right]$ & $24.2 \pm 6.0(17.4-43.3)$ \\
In a relationship & \\
Yes & $23(68 \%)$ \\
No & $11(32 \%)$ \\
Patella dislocation before surgery & \\
Less than 1 per year & $10(29 \%)$ \\
Annually & $5(15 \%)$ \\
Monthly & $8(24 \%)$ \\
Weekly & $5(15 \%)$ \\
Daily & $6(18 \%)$ \\
Patella re-dislocation after surgery & \\
No re-dislocation & $32(94 \%)$ \\
One re-dislocation & $1(3 \%)$ \\
Two or more re-dislocations & $1(3 \%)$ \\
\hline
\end{tabular}

Continuous variables are shown as mean \pm standard deviation (range), categorical variables are shown as number of patients and percentages of the total patient cohort in $77 \%$ of patients and combined MPFL-R $( \pm \mathrm{TTO})$ with two or more major bony procedures was performed in $24 \%$ (Table 2). Preoperatively, $57 \%$ of the total study group suffered from patellar dislocations at least monthly. Postoperatively, one patient suffered from one patellar re-dislocation and another patient from at least two patellar re-dislocations (re-dislocation rate: $6 \%$ ).

\section{Physical activity}

Results of physical activity outcome scores are summarized in Table 3. The median Tegner score improved from 3 to 4 , which was not statistically significant $(p=0.067)$. An improved sports ability was reported by $49 \%$ of patients

Table 2 Descriptive statistics of the surgical interventions of the total study group

\begin{tabular}{ll}
\hline Surgical interventions & Prevalence, $n(\%)$ \\
\hline MPFL-R $^{\S}+$ DFO & $18(53 \%)$ \\
MPFL-R* + Trochleoplasty & $7(21 \%)$ \\
MPFL-R* + HTO & $1(3 \%)$ \\
MPFL-R* + DFO+ Trochleoplasty & $6(18 \%)$ \\
MPFL-R + DFO+HTO+ Trochleoplasty & $1(3 \%)$ \\
MPFL-R + HTO + Trochleoplasty & $1(3 \%)$ \\
\hline
\end{tabular}

Categorical variables are shown as number of patients and percentages of the total patient cohort

$D F O$ distal femoral osteotomy, MPFL-R reconstruction of medial patellofemoral ligament with autologous gracilis tendon, HTO high tibial osteotomy, TTO tibial tubercle osteotomy

*Concomitant TTO was performed in 1 patient (3\%)

${ }^{\S}$ Concomitant TTO was performed in 2 patients $(6 \%)$

Table 3 Physical activity outcome scores of the total study group

\begin{tabular}{ll}
\hline & Total study group \\
\hline TEGNER current & $4 *$ (range 2-6) \\
TEGNER preoperative & $3 *$ (range 0-10) \\
Change in sports ability postoperatively & \\
No change, $n(\%)$ & $11(33 \%)$ \\
Improved, $n(\%)$ & $16(49 \%)$ \\
Deteriorated, $n(\%)$ & $6(18 \%)$ \\
Reasons for sports reduction postoperatively & \\
Knee joint complaints, $n(\%)$ & $6(55 \%)$ \\
Complaints in other parts of body, $n(\%)$ & $3(27 \%)$ \\
Other reasons (family, career, other interests), & $4(36 \%)$ \\
$n(\%)$ & \\
\hline
\end{tabular}

TEGNER Tegner activity scale

*Values are median; categorical variables are shown as number of patients and percentages of the total patient cohort

${ }^{\S}$ Question was answered by 33 patients

${ }^{\$}$ Multiple answers possible; affects 11 patients who have indicated that the sport frequency has been reduced postoperatively 
whereas $18 \%$ reported a decreased sports ability. Postoperatively, the most commonly performed sport activities were cycling (70\%), swimming (64\%), fitness (55\%), Nordic walking (30\%), hiking (30\%), and dancing (30\%).

\section{Quality of Life}

Mean EQ-VAS was $80.3 \pm 11.4$ (range 50-100) and mean EQ-5D-3L Index Value was $0.89 \pm 0.15$ (CI 0.84-0.94). Table 4 shows descriptive statistics of the five dimensions of QoL. Mean BPII 2.0 was $68.3 \pm 19.1$ (range 31-97). Significant correlations between results of EQ-VAS and BPII $2.0(r=0.725 ; p<0.001)$ and EQ-5D-3L Index value and BPII $2.0(r=0.638 ; p<0.001)$ could be determined.

\section{Sexual activity}

Results of the questionnaire regarding sexual activity are summarized in Table 5. In total, $34 \%$ of patients reported restrictions of sexual activities due to knee problems preoperatively, whereas $19 \%$ reported restrictions postoperatively. The most common reasons for preoperative restrictions of sexual activity were pain followed by apprehension. Of patients with preoperative restrictions, $60 \%$ reported improvement postoperatively.

\section{Discussion}

This study has several important findings. First, in young female patients with chronic PFI and anatomic risk factors, complex patellofemoral reconstruction with combined MPFL-R and bony procedures can reliably restore patellar stability, with a relatively low re-dislocation rate of $6 \%$. Second, despite the complexity and invasiveness of the procedures, sports ability could be improved in approximately $50 \%$ of patients. Third, QoL after complex patellofemoral reconstructions can be compared to QoL after isolated MPFL-R, as reported in the existing literature.

Table 4 Descriptive statistics of the five dimensions of Quality of Life with Euro-QoL-Score (EQ-5D-3L)

\begin{tabular}{llll}
\hline Dimension & Level 1 & Level 2 & Level 3 \\
\hline Mobility & $28(82 \%)$ & $6(18 \%)$ & \\
Self-care & $34(100 \%)$ & & \\
Usual activities & $31(91 \%)$ & $3(9 \%)$ & \\
Pain/discomfort & $14(41 \%)$ & $19(56 \%)$ & $1(3 \%)$ \\
Anxiety/depression & $26(77 \%)$ & $7(21 \%)$ & $1(3 \%)$ \\
\hline
\end{tabular}

Level 1: indicating no problem, Level 2: indicating some problems, Level 3: indicating extreme problems; categorical variables are shown as number of patients and percentages of the total patient cohort
No restrictions in self-care and only limited restrictions in usual activities and mobility could be found. Fourth, one third of female patients experienced restrictions in sexual activities due to problems associated with chronic PFI preoperatively. However, improved sexual activity was observed in $60 \%$ postoperatively.

The main intention of the present study was to describe the real-life benefit of comprehensive and invasive surgical procedures in this young patient cohort rather to describe score-based outcomes after a specific surgical procedure. Research after combined bony reconstructions and MPFL$\mathrm{R}$ in patients with PFI concentrated on functional outcomes so far [1, 13, 24, 26, 38], and important measures such as QoL have been largely neglected. In recent years, it has generally been recognized that QoL is an important outcome parameter after orthopedic interventions. Therefore, the BPII was specifically designed for patients with chronic PFI. However, current research on QoL in patients with PFI concentrated on outcomes after isolated MPFL-R only $[17-19,21]$. More recently, orthopedic studies have also focused on sexual activity outcomes [29, 33, 39, 40]. To the best of our knowledge, this aspect has not been investigated in patients with chronic PFI so far.

The present study focused on outcomes of female patients since young female patients have a higher risk of a primary and recurrent patellar dislocations [7, 11, 15] and tend to predispose to poorer functional outcomes [9, 25] as well as poorer QoL outcomes after MPFL-R [5, 17].

The re-dislocation rate has been considered a common outcome parameter of studies evaluating surgical treatment of chronic PFI. Comparable to our results, re-dislocation rate ranged from 0 to $5 \%$ in previous research after isolated MPFL-R [5, 9, 18, 21, 25, 44, 45]. Re-dislocation rate was $0 \%$ after derotational osteotomies of the distal femur and concomitant procedures (MPFL-R, patellofemoral arthroplasty, trochleoplasty, TTO) in patients with PFI during the follow-up period $[26,38]$ and comparably low after combined trochleoplasty and MPFL-R [1,24].

Since PFI results in decreased physical activity levels, physical activity remains an important outcome parameter postoperatively. In a systematic review, Schneider et al. reported a mean Tegner activity score of 5.7 points after isolated MPFL-R [45]. Several other authors reported similar results after isolated MPFL-R [4, 25]. In a study of Howells et al., $76 \%$ of subjects had resumed sporting activities at a mean follow-up of 16 months [25]. Comparable to the results of Imhoff et al. and Nelitz et al. after DFO and MPFL-R in patients with chronic PFI, mean Tegner activity scale in our cohort was lower [26, 38]. It can, therefore, be concluded that activity levels after complex patellofemoral reconstructions remain limited compared to isolated MPFL-R. Nevertheless, nearly half of subjects in 
Table 5 Descriptive statistics of sexual activity

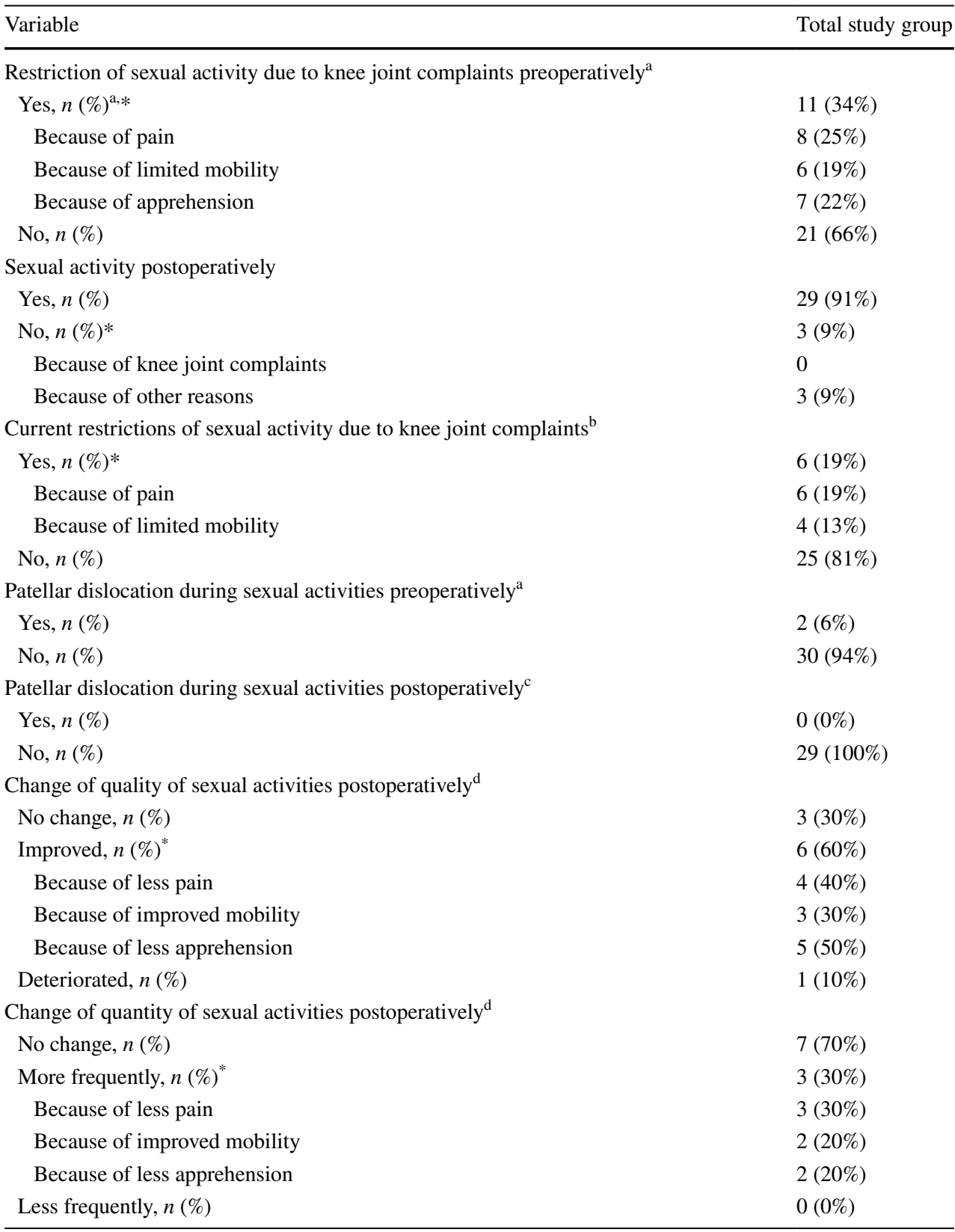

Categorical variables are shown as number of patients and percentages of patients

*Multiple answers possible

${ }^{\mathrm{a}} n=32$

${ }^{\mathrm{b}} n=31$

${ }^{\mathrm{c}} n=29$

${ }^{\mathrm{d}}$ Affects 11 patients who reported restrictions of sexual activities due to knee problems preoperatively, of those only 10 patients answered this question the present study claimed that physical activity improved postoperatively.

Another important finding of the present study was that QoL outcomes after complex reconstructions on the patellofemoral joint were comparable to the results after isolated MPFL-R or MPFL-R with concomitant TTO [17, 19, 21,
22]. Since the BPII is a patient-reported, disease-specific QoL score which is valid, reliable, and responsive in this patient population [20,31], we conclude that one or more additional bony intervention does not deteriorate the QoL outcomes in patients with chronic PFI. It must be noted, however, that QoL includes multidimensional aspects and 
can be affected by the patient's emotional and physical health, functional ability, and social environment [17]. Other studies assessed QoL after isolated MPFL-R with the EQ5D-3L score [4, 5]. After a comparable follow-up period, Biesert et al. described a mean EQ-5D-3L VAS of 70 and an EQ-5D-3L Index of 0.78 in a Swedish cohort [4]. After a shorter follow-up period, Bouras et al. found a mean EQ5D-3L VAS of 92 and an EQ-5D-3L Index of 1 in an English cohort [5]. Our results lie in between even though the surgical intervention was more extensive in our cohort. Compared to a representative survey in Germany [37], results of EQ-5D-3L VAS in our cohort were slightly below results of 25-44-year-old women in the mentioned study investigating health status in adults in Germany (80.3 versus 89). In the present study, psychological aspects, such as pain/ discomfort and anxiety/depression seemed to substantially influence QoL postoperatively. This assumption is further strengthened by previous research [5, 44]. A non-significant improvement in the anxiety and depression scores postoperatively was reported by Bouras et al. [5]. Even though the BPII is a disease-specific QoL outcome measure and the EQ-5D-3L a standardized generic measure of health status that can be applied to a wide range of health conditions, significant correlations between all QoL outcome scores could be found in the present study.

To the best of our knowledge, this is the first study to describe sexual activity outcomes after complex reconstructive knee surgery for chronic PFI. Sexual dysfunction shows a strong association with physical and emotional dissatisfaction and depression [32]. We therefore believe that improved sexual activity is an important goal after a complex reconstruction on the patellofemoral joint, especially in a young patient cohort. The percentage of patients sexually active postoperatively in our cohort was more than $90 \%$. This study demonstrated that most young female patients returned to their baseline or higher level of sexual activity after a complex surgical intervention because of PFI. Improvements in quality and frequency could be noted, caused by less pain, improved mobility, and less apprehension. In the life of a young women, difficulties during sexual activity can cause severe stress that could negatively influence QoL [33]. We, therefore, believe that it is important for an orthopedic surgeon to be able to make scientifically based statements on this subject in patient education, especially because young patients are affected.

This study has several limitations. First, this is a retrospective analysis without a control group. On the other hand, there are only few studies reporting on physical activity and QoL, and no study reporting on sexual activity after complex patellofemoral procedures. Second, the patient cohort is heterogenic concerning the surgical intervention, which is, however, attributable to the multifactorial pathogenesis of PFI. This can also be considered a strength, as the cohort is representative of the clinical situation. However, the main intention of the present study was not to describe the results of one specific procedure, but to describe physical activity, QoL, and sexual activity after invasive and complex patellofemoral reconstructions.

The results of this study have clinical relevance when considering postoperative outcomes, including physical and sexual activity and QoL, after complex patellofemoral reconstruction in patients suffering from chronic PFI. In the future, findings of this study can help to justify such comprehensive procedures and to improve preoperative patient counseling regarding postoperative expectations.

\section{Conclusion}

Complex patellofemoral reconstruction, defined as combined major bony procedures and MPFL-R plus/minus TTO in female patients suffering from chronic PFI leads to improved physical activity. QoL outcomes are comparable to outcomes after isolated MPFL-R as reported in the existing literature. Furthermore, this is the first study to describe limitations in sexual activity in female patients with PFI and that restrictions of sexual function can be limited postoperatively.

Author contributions MJF and PML designed the study. PML, PWW, MCR and SG collected data. MJF and PML performed the statistical analysis and wrote the manuscript. PWW, MCR and SG helped to design the study, assisted with statistical analysis and data interpretation, and critically reviewed the manuscript. ABI conceived of the study, helped with data interpretation and critically reviewed the manuscript. All authors read and approved the final manuscript.

Funding Open Access funding enabled and organized by Projekt DEAL.

\section{Compliance with ethical standards}

Conflict of interest Andreas B. Imhoff is a consultant for Arthrosurface and Medi Bayreuth and receives royalties from Arthrex and Arthrosurface. All other authors declare that they have no conflict of interest related to this study.

Ethical approval Ethical approval was obtained from the Ethics Committee of the technical University Munich. The study was approved by the institutional review board of the Technical University of Munich (575/19 S). All procedures performed were in accordance with the ethical standards of the institutional and/or national research committee and with the 1964 Declaration of Helsinki and its later amendments or comparable ethical standards.

Informed consent All subjects gave their written informed consent to participate in this investigation.

Open Access This article is licensed under a Creative Commons Attribution 4.0 International License, which permits use, sharing, adaptation, distribution and reproduction in any medium or format, as long 
as you give appropriate credit to the original author(s) and the source, provide a link to the Creative Commons licence, and indicate if changes were made. The images or other third party material in this article are included in the article's Creative Commons licence, unless indicated otherwise in a credit line to the material. If material is not included in the article's Creative Commons licence and your intended use is not permitted by statutory regulation or exceeds the permitted use, you will need to obtain permission directly from the copyright holder. To view a copy of this licence, visit http://creativecommons.org/licenses/by/4.0/.

\section{References}

1. Banke IJ, Kohn LM, Meidinger G, Otto A, Hensler D, Beitzel K et al (2014) Combined trochleoplasty and MPFL reconstruction for treatment of chronic patellofemoral instability: a prospective minimum 2-year follow-up study. Knee Surg Sports Traumatol Arthrosc 22:2591-2598

2. Becher C, Attal R, Balcarek P, Dirisamer F, Liebensteiner M, Pagenstert G et al (2018) Successful adaption of the Banff Patella Instability Instrument (BPII) 2.0 into German. Knee Surg Sports Traumatol Arthrosc 26:2679-2684

3. Becker R, Hirschmann MT, Karlsson J (2018) The complexity of patellofemoral instability. Knee Surg Sports Traumatol Arthrosc 26:675-676

4. Biesert M, Johansson A, Kostogiannis I, Roberts D (2020) Selfreported and performance-based outcomes following medial patellofemoral ligament reconstruction indicate successful improvements in knee stability after surgery despite remaining limitations in knee function. Knee Surg Sports Traumatol Arthrosc 28:934-940

5. Bouras T, Edmond U, Brown A, Gallacher P, Barnett A (2019) Isolated medial patellofemoral ligament reconstruction significantly improved quality of life in patients with recurrent patella dislocation. Knee Surg Sports Traumatol Arthrosc 27:3513-3517

6. Camathias C, Ammann E, Meier RL, Rutz E, Vavken P, Studer $\mathrm{K}$ (2020) Recurrent patellar dislocations in adolescents result in decreased knee flexion during the entire gait cycle. Knee Surg Sports Traumatol Arthrosc 28:2053-2066

7. Christensen TC, Sanders TL, Pareek A, Mohan R, Dahm DL, Krych AJ (2017) Risk factors and time to recurrent ipsilateral and contralateral patellar dislocations. Am J Sports Med 45:2105-2110

8. Dejour H, Walch G, Nove-Josserand L, Guier C (1994) Factors of patellar instability: an anatomic radiographic study. Knee Surg Sports Traumatol Arthrose 2:19-26

9. Enderlein D, Nielsen T, Christiansen SE, Faun $\varnothing$ P, Lind M (2014) Clinical outcome after reconstruction of the medial patellofemoral ligament in patients with recurrent patella instability. Knee Surg Sports Traumatol Arthrosc 22:2458-2464

10. Feucht MJ, Mehl J, Forkel P, Achtnich A, Schmitt A, Izadpanah $\mathrm{K}$ et al (2020) Failure analysis in patients with patellar redislocation after primary isolated medial patellofemoral ligament reconstruction. Orthop J Sports Med 8:2325967120926178

11. Fithian DC, Paxton EW, Stone ML, Silva P, Davis DK, Elias DA et al (2004) Epidemiology and natural history of acute patellar dislocation. Am J Sports Med 32:1114-1121

12. Flanigan DC, Shemory S, Lundy N, Stitgen M, Long JM, Magnussen RA (2020) Medial patellofemoral ligament reconstruction with allograft versus autograft tissue results in similar recurrent dislocation risk and patient-reported outcomes. Knee Surg Sports Traumatol Arthrosc 28:2099-2104

13. Frings J, Krause M, Akoto R, Wohlmuth P, Frosch K-H (2018) Combined distal femoral osteotomy (DFO) in genu valgum leads to reliable patellar stabilization and an improvement in knee function. Knee Surg Sports Traumatol Arthrosc 26:3572-3581

14. Gföller P, Hoser C, Runer A, Abermann E, Wierer G, Fink C (2019) Medial patellofemoral ligament (MPFL) reconstruction using quadriceps tendon autograft provides good clinical, functional and patient-reported outcome measurements (PROM): a 2-year prospective study. Knee Surg Sports Traumatol Arthrosc 27:2426-2432

15. Gravesen KS, Kallemose T, Blønd L, Troelsen A, Barfod KW (2018) High incidence of acute and recurrent patellar dislocations: a retrospective nationwide epidemiological study involving 24.154 primary dislocations. Knee Surg Sports Traumatol Arthrosc 26:1204-1209

16. Greiner W, Claes C, Busschbach J, von der Schulenburg J-MG (2005) Validating the EQ-5D with time trade off for the German population. Eur J Health Econ 6:124-130

17. Hiemstra LA, Kerslake S (2019) Age at time of surgery but not sex is related to outcomes after medial patellofemoral ligament reconstruction. Am J Sports Med 47:1638-1644

18. Hiemstra LA, Kerslake S, Kupfer N, Lafave M (2019) Patellofemoral stabilization: postoperative redislocation and risk factors following surgery. Orthop J Sports Med 7:2325967119852627

19. Hiemstra LA, Kerslake S, Lafave MR (2017) Quality-of-life outcomes of patients following patellofemoral stabilization surgery: the influence of trochlear dysplasia. J Knee Surg 30:887-893

20. Hiemstra LA, Kerslake S, Lafave MR, Heard SM, Buchko GM, Mohtadi NG (2013) Initial validity and reliability of the Banff Patella Instability Instrument. Am J Sports Med 41:1629-1635

21. Hiemstra LA, Kerslake S, Lafave MR, Tucker A (2020) Patella alta is reduced following MPFL reconstruction but has no effect on quality-of-life outcomes in patients with patellofemoral instability. Knee Surg Sports Traumatol Arthrosc. https://doi. org/10.1007/s00167-020-05977-8

22. Hiemstra LA, Kerslake S, Loewen M, Lafave M (2016) Effect of trochlear dysplasia on outcomes after isolated soft tissue stabilization for patellar instability. Am J Sports Med 44:1515-1523

23. Hiemstra LA, Page JL, Kerslake S (2019) Patient-reported outcome measures for patellofemoral instability: a critical review. Curr Rev Musculoskelet Med 12:124-137

24. Hiemstra LA, Peterson D, Youssef M, Soliman J, Banfield L, Ayeni OR (2019) Trochleoplasty provides good clinical outcomes and an acceptable complication profile in both short and long-term follow-up. Knee Surg Sports Traumatol Arthrosc 27:2967-2983

25. Howells N, Barnett A, Ahearn N, Ansari A, Eldridge J (2012) Medial patellofemoral ligament reconstruction: a prospective outcome assessment of a large single centre series. J Bone Joint Surg Br 94:1202-1208

26. Imhoff FB, Cotic M, Liska F, Dyrna FGE, Beitzel K, Imhoff AB et al (2019) Derotational osteotomy at the distal femur is effective to treat patients with patellar instability. Knee Surg Sports Traumatol Arthrosc 27:652-658

27. Imhoff FB, Funke V, Muench LN, Sauter A, Englmaier M, Woertler K et al (2020) The complexity of bony malalignment in patellofemoral disorders: femoral and tibial torsion, trochlear dysplasia, TT-TG distance, and frontal mechanical axis correlate with each other. Knee Surg Sports Traumatol Arthrosc 28:897-904

28. Kaiser P, Schmoelz W, Schöttle P, Heinrichs C, Zwierzina M, Attal R (2019) Isolated medial patellofemoral ligament reconstruction for patella instability is insufficient for higher degrees of internal femoral torsion. Knee Surg Sports Traumatol Arthrosc 27:758-765

29. Kazarian GS, Lonner JH, Hozack WJ, Woodward L, Chen AF (2017) Improvements in sexual activity after total knee arthroplasty. J Arthroplasty 32:1159-1163 
30. Krych AJ, O’Malley MP, Johnson NR, Mohan R, Hewett TE, Stuart MJ et al (2018) Functional testing and return to sport following stabilization surgery for recurrent lateral patellar instability in competitive athletes. Knee Surg Sports Traumatol Arthrosc 26:711-718

31. Lafave MR, Hiemstra L, Kerslake S (2016) Factor Analysis and Item Reduction of the Banff Patella Instability Instrument (BPII): introduction of BPII 2.0. Am J Sports Med 44:2081-2086

32. Laumann EO, Paik A, Rosen RC (1999) Sexual dysfunction in the United States: prevalence and predictors. JAMA 281:537-544

33. Lee S, Frank RM, Harris J, Song SH, Bush-Joseph CA, Salata MJ et al (2015) Evaluation of sexual function before and after hip arthroscopic surgery for symptomatic femoroacetabular impingement. Am J Sports Med 43:1850-1856

34. Lewallen LW, McIntosh AL, Dahm DL (2013) Predictors of recurrent instability after acute patellofemoral dislocation in pediatric and adolescent patients. Am J Sports Med 41:575-581

35. Lind M, Enderlein D, Nielsen T, Christiansen SE, Faunø P (2016) Clinical outcome after reconstruction of the medial patellofemoral ligament in paediatric patients with recurrent patella instability. Knee Surg Sports Traumatol Arthrosc 24:666-671

36. Ménétrey J, Putman S, Gard S (2014) Return to sport after patellar dislocation or following surgery for patellofemoral instability. Knee Surg Sports Traumatol Arthrosc 22:2320-2326

37. Mielck A, Vogelmann M, Schweikert B, Leidl R (2010) Gesundheitszustand bei Erwachsenen in Deutschland: Ergebnisse einer repräsentativen Befragung mit dem EuroQol 5D (EQ-5D). Gesundheitswesen 72:476-486

38. Nelitz M, Dreyhaupt J, Williams SRM, Dornacher D (2015) Combined supracondylar femoral derotation osteotomy and patellofemoral ligament reconstruction for recurrent patellar dislocation and severe femoral anteversion syndrome: surgical technique and clinical outcome. Int Orthop 39:2355-2362

39. Nugent RE, Cheesman QT, Bradian AK, Austin LS (2020) Improvements in sexual function following arthroscopic rotator cuff repair. J Shoulder Elbow Surg. https://doi.org/10.1016/j. jse.2020.06.017

40. Nunley RM, Nam D, Bashyal RK, Della Valle CJ, Hamilton WG, Berend ME et al (2015) The impact of total joint arthroplasty on sexual function in young, active patients. J Arthroplasty $30: 335-340$

41. Oppe M, Devlin NJ, Szende A (2007) EQ-5D value sets: inventory, comparative review and user guide. ISBN-13 978-1-40205511-9 (e-book). https://doi.org/10.1007/1-4020-5511-0

42. Rabin R, Charro Fd (2001) EQ-SD: a measure of health status from the EuroQol Group. Ann Med 33:337-343

43. Sanders TL, Pareek A, Hewett TE, Stuart MJ, Dahm DL, Krych AJ (2018) High rate of recurrent patellar dislocation in skeletally immature patients: a long-term population-based study. Knee Surg Sports Traumatol Arthrosc 26:1037-1043

44. Sappey-Marinier E, Sonnery-Cottet B, O'Loughlin P, Ouanezar H, Reina Fernandes L, Kouevidjin B et al (2019) Clinical outcomes and predictive factors for failure with isolated MPFL reconstruction for recurrent patellar instability: a series of 211 reconstructions with a minimum follow-up of 3 years. Am J Sports Med 47:1323-1330

45. Schneider DK, Grawe B, Magnussen RA, Ceasar A, Parikh SN, Wall EJ et al (2016) Outcomes after isolated medial patellofemoral ligament reconstruction for the treatment of recurrent lateral patellar dislocations: a systematic review and meta-analysis. Am J Sports Med 44:2993-3005

46. Senavongse W, Amis A (2005) The effects of articular, retinacular, or muscular deficiencies on patellofemoral joint stability: a biomechanical study in vitro. J Bone Joint Surg Br 87:577-582

47. Stephen JM, Lumpaopong P, Dodds AL, Williams A, Amis AA (2015) The effect of tibial tuberosity medialization and lateralization on patellofemoral joint kinematics, contact mechanics, and stability. Am J Sports Med 43:186-194

48. Tegner Y, Lysholm J (1985) Rating systems in the evaluation of knee ligament injuries. Clin Orthop Relat Res 198:43-49

49. Thomas L (1997) Retrospective power analysis. Conserv Biol 11:276-280

Publisher's Note Springer Nature remains neutral with regard to jurisdictional claims in published maps and institutional affiliations. 